\title{
Lower and Upper Bounds for Scaling Factors Used for Integer Approximation of 3D Anisotropic Chamfer Distance Operator
}

\author{
Didier Coquin and Philippe Bolon \\ LISTIC, Domaine Universitaire, BP 80439, 74944 Annecy le Vieux Cedex, France \\ didier.coquin@univ-savoie.fr
}

\begin{abstract}
For 3D images composed of successive scanner slices (e.g. medical imaging, confocal microscopy or computed tomography), the sampling step may vary according to the axes, and specially according to the depth which can take values lower or higher than 1 . Hence, the sampling grid turns out to be parallelepipedic. In this paper, 3D anisotropic local distance operators are introduced. The problem of coefficient optimization is addressed for arbitrary mask size. Lower and upper bounds of scaling factors used for integer approximation are given. This allows, first, to derive analytically the maximal normalized error with respect to Euclidean distance, in any 3D anisotropic lattice, and second, to compute optimal chamfer coefficients. As far as large images or volumes are concerned, 3D anisotropic operators are adapted to the measurement of distances between objects sampled on non-cubic grids as well as for quantitative comparison between grey level images.
\end{abstract}

Keywords: Distance transformation, Chamfer distance, Anisotropic lattice.

\section{Introduction}

In image analysis, measuring distances between objects is often essential. The notion of distance is very useful to describe a pattern in a digital image or to characterize the location of objects inside a volume. This is useful in many different shape representations and shape recognition tasks. An overview of various applications can be found in [1|2|3]. The aim of a distance transformation is to compute the distance from a point to an object, i.e. to a set of points. The distance from point $p$ to the object is the smallest distance from $p$ to any point of the object. In other words, it is the distance from $p$ to the nearest point $q$ belonging to the object. The two main approaches to the computation of distance maps are Chamfer Distance operators [1] and Euclidean Distance operators [45]. In the first case, the distance between two pixels is defined as the length of the minimal path between them. This path is composed of a finite sequence of elementary steps to which weights are assigned. Distance maps are obtained by propagating local distances. In most cases, they do not yield the exact Euclidean distance between pixels. In the latter case, quasi-exact or exact Euclidean distances are obtained by taking the relative location between pixels into account. Interesting analysis are presented in [6778].

S. Brlek, C. Reutenauer, and X. Provençal (Eds.): DGCI 2009, LNCS 5810, pp. 457-468, 2009.

(C) Springer-Verlag Berlin Heidelberg 2009 
To improve Chamfer Distance Transforms, efforts have been made in four directions:

(i) decreasing the sensitivity to rotation by means of a better approximation of the Euclidean distance. This is achieved by assigning weights to elementary displacements allowed by the local distance operator. These weights are optimized according to an error criterion which generally consists in minimizing the maximum difference between the computed distance and the Euclidean distance along a reference trajectory, which could be rectilinear [3] or circular [1011].

(ii) increasing the dimension of the image space. Weighted distance transforms in 3D were introduced as early as 1984 [2]. Different approaches are possible to set the local coefficients, based either on a discrete model [12 13] or a continuous one [10 1114]. Reference trajectories may be rectilinear [14] or spherical [10]. Coefficients for $5 \times 5 \times 5$ local operators were proposed in [6 10 12 18]. Higher dimension distance transforms were used for skeletonisation in 4D space [15]. However, some practical difficulties may arise because of memory size requirements.

(iii) studying generic properties. Whether distances computed by means of distance transforms are metric or not may be of great interest for optimization purposes. Semiregularity conditions state that any discrete path composed of repetitions of elementary displacements is optimal. In [16], Kiselman showed that a distance transform in $\mathbf{Z}^{n}$ producing a discrete norm is semi-regular. Moreover, such a semi-regularity distance is metric in $\mathbf{Z}^{2}$. Conditions for obtaining a discrete norm with a $5 \times 5 \times 5$ operator are given in [12]. Metricity is considered in [13].

(iv) adapting the local operator to non cubic sampling grids. Imaging systems often produce images having different sampling steps according to the different axes. In most cases, the resulting image is composed of parallelepipedic voxels having two sides equal and the third different. For computed tomography, or confocal microscopy, the ratio between the largest to the shortest voxel dimension typically ranges from 1 to 10 [1719]. Another way to decrease the maximum error between the Euclidean distance and the local distance is to increase the size of the mask operator. Since voxels may not be cubic, anisotropic masks have to be considered.

In this paper, the general case of $\mathrm{MxMxV}$ distance operators adapted to parallelepipedic grids composed of voxels having a WxWxP size is addressed. These so-called anisotropic operators yield the same performances as those of isotropic ones (MxMxM) with reduced complexity and computation time. In Section 2, optimal isotropic operators adapted to anisotropic grids are introduced. The influence of voxel depth $\mathrm{P}$ and operator size $\mathrm{M}$ is studied. Section 3 addresses a new issue of integer approximation implementation, by choosing a scaling factor between lower and upper bounds. Examples of integer chamfer masks are given.

\section{3D Anisotropic Chamfer Distance Operator}

The objective is to approximate the real Euclidean distance $d_{E}$. The optimization criterion consists in minimizing the maximum error between the local distance $d_{L}$ and the Euclidean distance $d_{E}$. The maximum error is sometimes called the maximum absolute error. In both cases, it is the absolute value of the maximum difference between the chamfer local distance $d_{L}$ and the Euclidean distance $d_{E}$. In [20] we have proposed a 
generalization to parallelepipedic grids of the approach developed by Verwer [10]. But unlike the approach followed by Verwer which directly gives the position and the amplitude of the maximum absolute error, our method allows error analysis at any point of the sphere. Thus we have a better control of optimization, especially in the case of the integer approximation which generally involves a displacement of the maximum absolute error location.

Given a grid, what is the optimal mask size ? In this paper, we consider the case of voxels having two sides equal (width $\mathrm{W}$ and height $\mathrm{H}$ ) and the third different (depth $\mathrm{P})$. This is the situation with most of $3 \mathrm{D}$ images such as those obtained by confocal microscopy or computed tomography [7]. It is also the case for grey level images where geometrical distortions (spatial domain) and radiometric distortions (grey level domain) are assigned different weights.

The directions in which voxels have the same size may be referred to as "horizontal" dimensions whereas the other directions may be referred to as "vertical" dimensions.

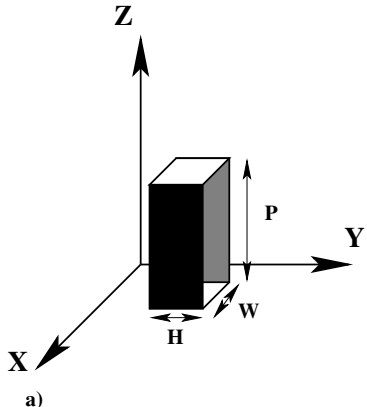

a)

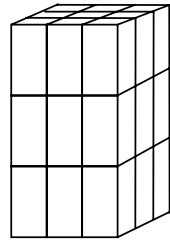

b)

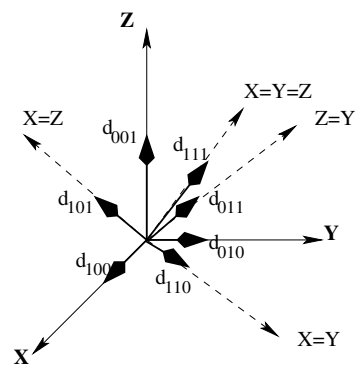

c)

Fig. 1. a) Voxel's characteristic, b) cubic $3 \times 3 \times 3$ operator, c) elementary displacements $d_{i j k}$ for $3 \times 3 \times 3$ mask

Local distance operators are characterized by the coefficients assigned to the elementary displacements. For a cubic MxMxM operator, with $\mathrm{M}=2 \mathrm{~m}+1$, one needs to set coefficients $d_{i j k}$ with $i, j, k \in\{0,1,2, \ldots, m\}$ and $\operatorname{GCD}(\mathrm{i}, \mathrm{j}, \mathrm{k})=1, i$ and $j$ denote the number of steps in horizontal dimensions, $k$ denotes the number of steps in the vertical dimension. The directions of the elementary displacements $d_{i j k}$ are shown in Figure 1, for a cubic $3 \times 3 \times 3$ operator $(M=3$, and $m=1)$. They define parts in the image space. We assume that $W \leq P$.

Let voxel $\mathrm{O}$ be the origin of a binary digitized $3 \mathrm{D}$ image. Let $\mathrm{x}, \mathrm{y}$ and $\mathrm{z}$ be the coordinates in the image referential, and (OX,OY,OZ) the actual space referential. Let voxel $\mathrm{Q}(\mathrm{x}, \mathrm{y}, \mathrm{z})$ describe a sphere having fixed radius $\mathrm{R}$. The equation of the trajectory is $d_{E}=O Q=\sqrt{(W x)^{2}+(W y)^{2}+(P z)^{2}}=R$. We assume that the fixed value of $\mathrm{R}$ is large with respect to $\mathrm{W}$ and $\mathrm{P}$, so that displacements between two adjacent voxels can be regarded as continuous.

Verwer [10] showed that the maximum error between $d_{E}$ and $d_{L}$ occurs in the cone having the greatest angle. For a cubic $\mathrm{MxMxM}$ operator $(\mathrm{M}=2 \mathrm{~m}+1)$, the maximum error between the local distance $d_{L}$ and the Euclidean distance $d_{E}$ occurs in the part limited by the directions of $d_{100}, d_{m 10}$ and $d_{m 11}$. 
The values of the maximum error $e_{\max }$ (normalized by radius $R$ ) produced by using a 3D cubic operator of different sizes, when $W=H=P=1$, are summarized in Table 1 It should be noticed that $e_{\max }$ decreases with the mask size [2]10].

Table 1. Maximal normalized error produced with isotropic operator in $3 \mathrm{D}(W=H=P=1)$

\begin{tabular}{|c||c|c|c|c|c|}
\hline mask size & $3 \times 3 \times 3$ & $5 \times 5 \times 5$ & $7 \times 7 \times 7$ & $9 \times 9 \times 9$ & $11 \times 11 \times 11$ \\
\hline$e_{\max } \%$ & 6.019 & 2.411 & 1.223 & 0.725 & 0.476 \\
\hline
\end{tabular}

The study of the error evolution $e_{\max }$ as a function of depth $P$ shows that the larger $P$ is, the larger the maximum error occurring in the part limited by the directions of $d_{100}, d_{m 10}$ and $d_{m 11}$. By using non-cubic masks (mask of size MxMxV) it is possible to reduce the computation time and still to achieve the accuracy [20].

\subsection{Non-cubic Operator Optimization in 3D}

With parallelepipedic grids, voxels are characterized by their width $\mathrm{W}$, their height $\mathrm{H}$, and their depth $\mathrm{P}$ that are generally different. Since horizontal voxel dimensions are equal $(W=H)$, two cases have to be considered.

- First case $P \geq 1$ : the maximum error occurs in the first part of the first octant of the sphere (limited by the directions of $d_{100}, d_{m 10}$ and $d_{m 11}$ ) and increases with $P$. The maximum absolute error decreases with the mask size [20]. We then choose a mask MxMxV with $M \geq V(M=2 m+1$ and $V=2 v+1)$. Figure 2 presents the mask of a $5 \times 5 \times 3$ operator, and the different part for optimizations.

- Second case $0<P \leq 1$ : the maximum error occurs in the last part of the first octant of the sphere (limited by the directions of $d_{001}, d_{01 v}$ and $d_{11 v}$ ) and decreases with $P$. The maximum absolute error decreases with the mask size. We then choose a mask MxMxV with $M \leq V(M=2 m+1$ and $V=2 v+1)$. Figure 3 presents the mask of a $3 \times 3 \times 5$ operator, and the different parts for the optimizations.

Coefficient optimization: Two procedures are available:

(1) Minimizing the error in the first part limited by the directions of $d_{100}, d_{m 10}$ and $d_{m 11}$ (Fig.2b). The local distance in this part is given by:

$$
d_{L}(O, Q)=d_{100} \cdot x+\left(d_{m 10}-m \cdot d_{100}\right) \cdot y+\left(d_{m 11}-d_{m 10}\right) \cdot z
$$

It can be expressed as a function of $y$ and $z$ :

$d_{L}(y, z)=\frac{d_{100}}{W} \cdot \sqrt{(R)^{2}-(W y)^{2}-(P z)^{2}}+\left(d_{m 10}-m \cdot d_{100}\right) \cdot y+\left(d_{m 11}-d_{m 10}\right) \cdot z$

Hence, the error $E(y, z)=d_{L}-d_{E}$ is:

$$
E(y, z)=\frac{d_{100}}{W} \cdot \sqrt{(R)^{2}-(W y)^{2}-(P z)^{2}}+\left(d_{m 10}-m \cdot d_{100}\right) \cdot y+\left(d_{m 11}-d_{m 10}\right) \cdot z-R
$$




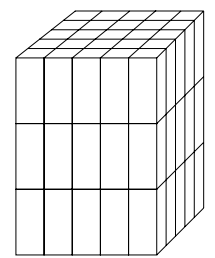

a)
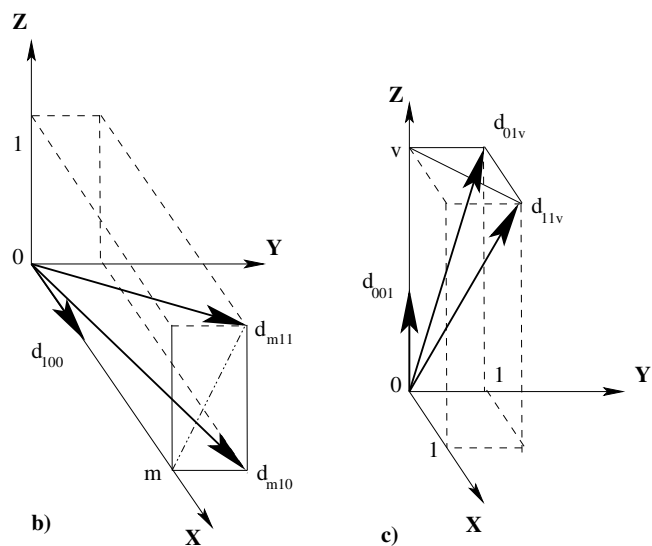

Fig. 2. Operator $M x M x V$ in parallelepipedic grid, a) $5 \times 5 \times 3$ operator, b) first part of the first octant of the sphere, c) last part of the first octant of the sphere.

$E(y, z)$ is extremal at the interval borders and when the partial first derivatives are zero. The maximum absolute normalized error $e_{1}^{\prime}$ is:

$$
e_{1}^{\prime}=\frac{E_{\max }}{R}=\left|1-\frac{d_{100}}{W}\right|
$$

with

$$
d_{100}=\frac{-2 W+2 W \sqrt{1+\lambda_{1}^{\prime}}}{\lambda_{1}^{\prime}}
$$

and

$$
\lambda_{1}^{\prime}=\frac{1}{W^{2}}\left(T_{m 10}-m W\right)^{2}+\frac{1}{P^{2}}\left(T_{m 11}-T_{m 10}\right)^{2}
$$

Since $W=H=1$, the error depends on $P$ and $m$. Coefficients $d_{i j k}$ are given by:

$$
d_{i j k}=T_{i j k} \frac{d_{100}}{W}
$$

with

$$
T_{i j k}=\sqrt{(i W)^{2}+(j W)^{2}+(k P)^{2}}
$$

(2) Minimizing the error in the last part limited by the directions of $d_{001}, d_{01 v}$ and $d_{11 v}$ (Fig. 2, yields:

$$
d_{001}=\frac{-2 P+2 P \sqrt{1+\lambda_{2}^{\prime}}}{\lambda_{2}^{\prime}}
$$

with

$$
\lambda_{2}^{\prime}=\frac{1}{W^{2}}\left(T_{11 v}-T_{01 v}\right)^{2}+\frac{1}{W^{2}}\left(T_{01 v}-v P\right)^{2}
$$

Coefficients $d_{i j k}$ are given by:

$$
d_{i j k}=T_{i j k} \frac{d_{001}}{P}
$$




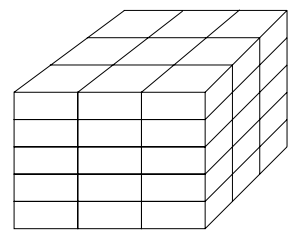

a)

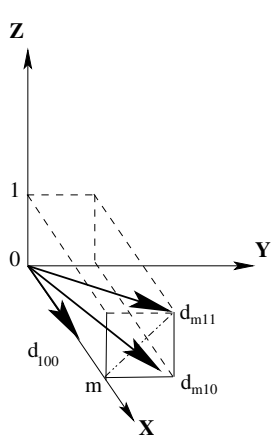

b)

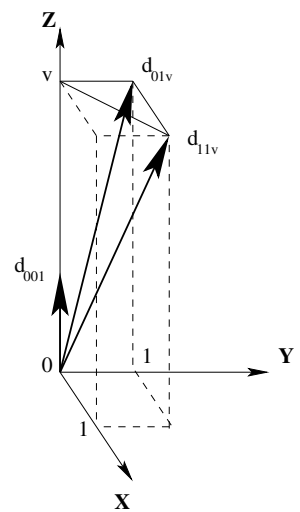

Fig. 3. Operator $\mathrm{MxMxV}$ in parallelepipedic grid,a) $3 \times 3 \times 5$ operator, b) first part of the first octant of the sphere, c) last part of the first octant of the sphere

with

$$
T_{i j k}=\sqrt{(i W)^{2}+(j W)^{2}+(k P)^{2}}
$$

the maximum absolute normalized error $e_{2}^{\prime}$ is:

$$
e_{2}^{\prime}=1-\frac{d_{001}}{P}
$$

Since $W=H=1$, the error depends on $P$ and $v$.

For $P \geq 1$, the study of $e_{1}^{\prime}$ and $e_{2}^{\prime}$ as functions of voxel depth $P$ yields that $e_{1}^{\prime}$ is steadily increasing function of $\mathrm{P}$ whereas $e_{2}^{\prime}$ is a steadily decreasing function. We have $e_{1}^{\prime}=e_{2}^{\prime}$ for $P=P_{0}$. Let $P_{0}$ be the solution to the equation $\lambda_{1}^{\prime}=\lambda_{2}^{\prime}$.

- if $P \leq P_{0}$ : the second optimization procedure must be performed. Coefficients are given by eq 11 The maximum absolute normalized error is $e_{\max }=e_{2}^{\prime}$.

- if $P \geq P_{0}$ : the first optimization procedure must be performed. Coefficients are given by eq7 The maximum absolute normalized error is $e_{\max }=e_{1}^{\prime}$.

For $0<P \leq 1$, the study of $e_{1}^{\prime}$ and $e_{2}^{\prime}$ as functions of voxel depth $P$ yields that $e_{1}^{\prime}$ is steadily increasing function of $\mathrm{P}$ whereas $e_{2}^{\prime}$ is a steadily decreasing function. We have $e_{1}^{\prime}=e_{2}^{\prime}$ for $P=P_{0}^{\prime}$. Let $P_{0}^{\prime}$ be the solution to the equation $\lambda_{1}^{\prime}=\lambda_{2}^{\prime}$.

- if $P \leq P_{0}^{\prime} \leq 1$ : the second optimization procedure must be performed. Coefficients are given by eq 11 . The maximum absolute normalized error is $e_{\max }=e_{2}^{\prime}$.

- if $1 \geq P \geq P_{0}^{\prime}$ : the first optimization procedure must be performed. Coefficients are given by eq77 The maximum absolute normalized error is $e_{\max }=e_{1}^{\prime}$.

\subsection{Performance Study}

In this subsection, we study the performances of 3D anisotropic operators. Some examples of $3 \mathrm{D}$ operators of different dimensions are given. 
The values of the maximum normalized error produced by using $3 \mathrm{D}$ operators of different sizes, when voxel dimensions are $W=H=1$ and $P=2$, are summarized in Table 2. It can be noticed that isotropic operators can be replaced by anisotropic ones (smaller in size) without reducing the performances (value of $e_{\max }$ ).

Table 2. Maximal normalized error produced with 3D anisotropic operators for $W=H=$ $1, P=2$

\begin{tabular}{|c||c|c|c|c|c|}
\hline mask size & $3 \times 3 \times 3$ & $5 \times 5 \times 5$ & $7 \times 7 \times 7$ & $9 \times 9 \times 9$ & $5 \times 5 \times 3$ \\
\hline$e_{\max } \%$ & 9.08 & 4.59 & 2.61 & 1.64 & 4.59 \\
\hline mask size & $7 \times 7 \times 3$ & $9 \times 9 \times 3$ & $11 \times 11 \times 3$ & $9 \times 9 \times 5$ & $11 \times 11 \times 5$ \\
\hline$e_{\max } \%$ & 2.61 & 2.41 & 2.41 & 1.64 & 1.11 \\
\hline
\end{tabular}

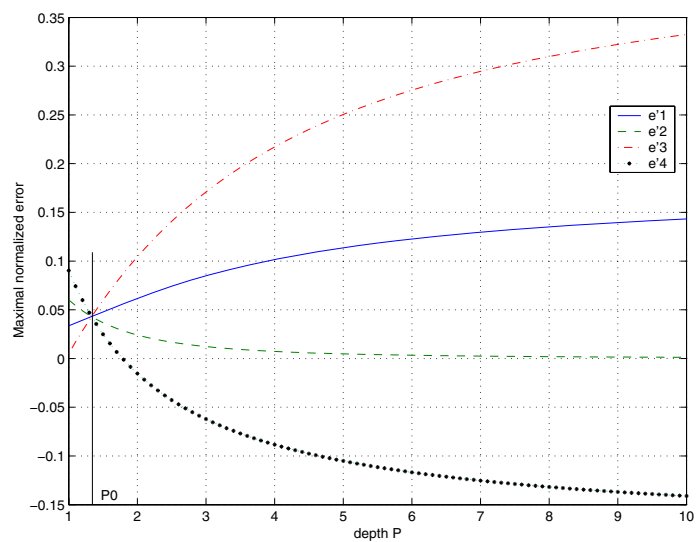

Fig. 4. Normalized error versus depth $P \geq 1$ for $5 \times 5 \times 3$ operator

The error functions $e_{1}^{\prime}$ and $e_{2}^{\prime}$ produced by a $5 \times 5 \times 3$ operator with the two optimization procedures given in Section 2.1, are shown in Figure 4 In this case, $P_{0}=1.54$. It is the solution to the equation $\lambda_{1}^{\prime}=\lambda_{2}^{\prime}$. The first optimization is better for $P \geq P_{0}$, and the second one for $P \leq P_{0}$. Curve $e_{3}$ represents the maximum error in the last part of the sphere with the first optimization, and curve $e_{4}^{\prime}$ gives the maximum error in the first part of the sphere with the second optimization. Curve $e_{2}^{\prime}$ in dashed green line for $P \leq P_{0}$ and curve $e_{1}^{\prime}$ in solid blue line for $P \geq P_{0}$ represent the maximum absolute normalized error obtained.

The error functions $e_{1}^{\prime}$ and $e_{2}^{\prime}$ produced by a $3 \times 3 \times 5$ operator with the two optimization procedures given in 3.1 are shown in Figure 5 In this case $P_{0}^{\prime}=0.61$. It is the solution to the equation $\lambda_{1}^{\prime}=\lambda_{2}^{\prime}$. The first optimization is better for $P \geq P_{0}^{\prime}$, and the second one for $P \leq P_{0}^{\prime}$. Curve $e_{2}^{\prime}$ in a dashed green line for $P \leq P_{0}^{\prime}$ and curve $e_{1}^{\prime}$ in a solid blue line for $\bar{P} \geq P_{0}^{\prime}$ represent the maximum absolute normalized errors obtained. 


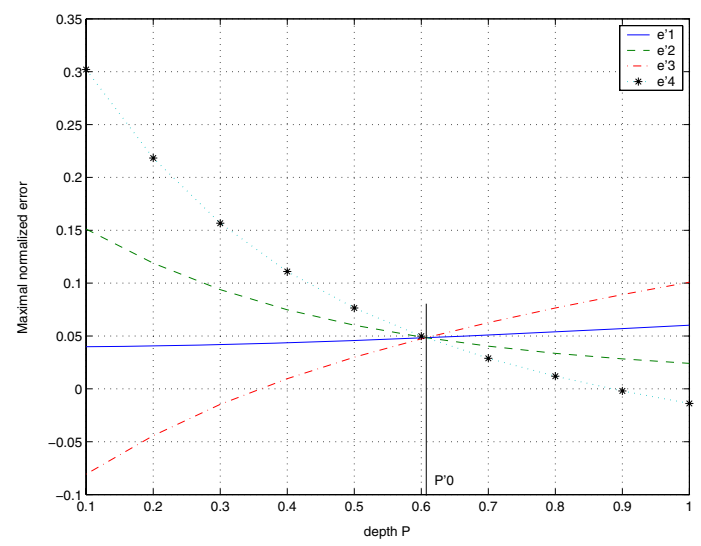

Fig. 5. Normalized error versus depth $0<P \leq 1$ for $3 \times 3 \times 5$ operator

\section{Integer Approximation and Implementation}

Because of time and memory constraints, it may be preferable to use operators with integer coefficients. The integer approximation is achieved by multiplying the real coefficients by an integer $N$ and rounding to the nearest integer. In this section we propose a lower bound $\left(N_{\min }\right)$ aiming at preserving the distance transform accuracy with respect to the Euclidean distance, and an upper bound $\left(N_{\max }\right)$ that guarantees that there is no numerical overflow in the distance representation.

Let $i$ be the number of bits needed to encode the distance value at each voxel. Let $\mathrm{Dim}$ be the image dimension. The maximum coded distance is

$$
D_{\max }=\operatorname{Dim} \cdot \operatorname{round}\left(N \cdot d_{111}\right)=\operatorname{Dim} \cdot\left(N \cdot d_{111}+q\right)
$$

where $q$ is the rounding error such that $|q| \leq \frac{1}{2}$

For large $N$, the rounding error can be neglected, so that we have:

$$
N_{\max }<\frac{2^{i}}{\operatorname{Dim} \cdot d_{111}}
$$

where

$$
d_{111}=\left(\sqrt{2+P^{2}}\right)\left[\frac{-2+2 \sqrt{1+\lambda}}{\lambda}\right]
$$

and

$$
\lambda=\left(\sqrt{\left(m^{2}+1\right)}-m\right)^{2}+\frac{1}{P^{2}}\left[\sqrt{\left(m^{2}+1+P^{2}\right)}-\sqrt{\left(m^{2}+1\right)}\right]^{2}
$$

where $M=2 m+1$ is the operator mask size.

The minimum value is chosen so that the rounding error is of the same order of magnitude as that of the distance transform.

Let $E_{\max }$ be the maximum error of the distance transform. As shown in [10], this maximum error is proportional to the distance $R$. It is obtained in the center of the 
first cone and in direction $d_{100}$. Let $D_{100}$ be the integer representation of elementary displacement $d_{100}$. We have

$$
D_{100}=\operatorname{round}\left(N \cdot d_{100}\right)
$$

and the relative error induced by the integer approximation is

$$
|\epsilon|=\frac{\left|\operatorname{round}\left(N \cdot d_{100}\right)-N \cdot d_{100}\right|}{N \cdot d_{100}}=\frac{|q|}{N \cdot d_{100}}=\frac{1}{2 . N \cdot d_{100}}
$$

with $|q| \leq \frac{1}{2}$

For $W=H=1$, the maximum absolute normalized error induced by using a local distance operator is:

$$
\left|e_{\max }\right|=\frac{\left|E_{\max }\right|}{R}=\left|1-d_{100}\right|
$$

By taking eq20 and eq5into account, the minimum scaling factor value must satisfy

$$
N_{\min }>\frac{1}{2 . d_{100} \cdot e_{\max }}
$$

where

$$
d_{100}=\frac{-2+2 \sqrt{1+\lambda}}{\lambda}
$$

and $\lambda$ is given by eq 17 It can be seen that $N_{\min }$ depends on voxel depth $P$.

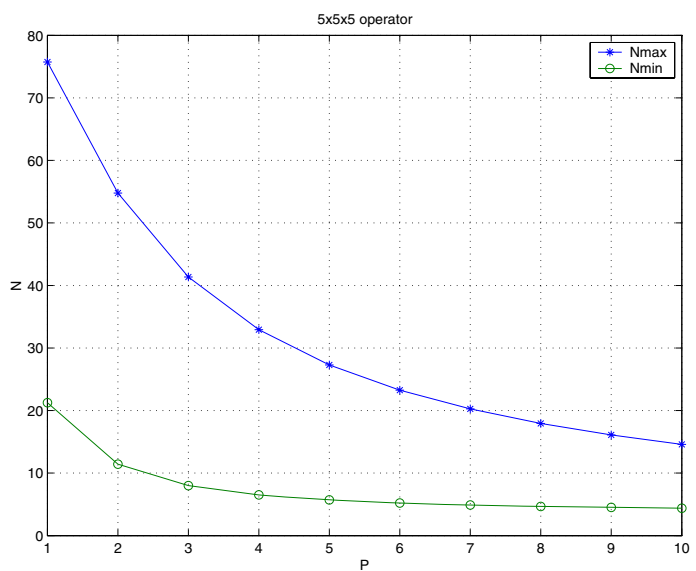

Fig. 6. Scaling factor bounds $N_{\max }$ and $N_{\min }$ for a $5 \times 5 \times 5$ operator versus voxel depth $P$

It should be noticed that eq 15 is valid if the volume dimensions are the same in rows, columns and layers. For eq 18 , it is assumed that the real coefficients are set to their optimum value. 


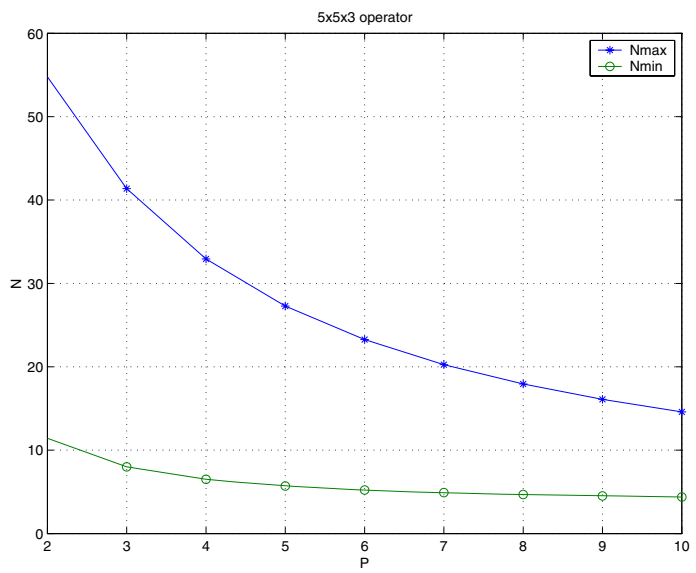

Fig. 7. Scaling factor bounds $N_{\max }$ and $N_{\min }$ for a $5 \times 5 \times 3$ operator versus voxel depth $P$

Table 3. Best integer approximation of some isotropic $W=H=P=1$ and anisotropic operators in $3 \mathrm{D}$ with and $W=H=1, P=2, i=16$ bits, $\mathrm{Dim}=512$

\begin{tabular}{|c||c|c||c|c||}
\hline $\mathrm{W}, \mathrm{H}, \mathrm{P}$ & $(1,1,1)$ & $(1,1,1)$ & $(1,1,2)$ & $(1,1,2)$ \\
\hline size & $3 \times 3 \times 3$ & $5 \times 5 \times 5$ & $5 \times 5 \times 5$ & $5 \times 5 \times 3$ \\
\hline$N_{\min }$ & 8.84 & 21.25 & 11.42 & 11.42 \\
\hline$N_{\max }$ & 78.63 & 75.72 & 54.77 & 54.77 \\
\hline$N$ & 67 & 43 & 22 & 44 \\
\hline$D_{100}, D_{010}$ & 63 & 42 & 21 & 42 \\
\hline$D_{001}$ & 63 & 42 & 42 & 84 \\
\hline$D_{110}$ & 89 & 59 & 30 & 59 \\
\hline$D_{101}, D_{011}$ & 89 & 59 & 47 & 94 \\
\hline$D_{111}$ & 109 & 73 & 51 & 103 \\
\hline$D_{210}, D_{120}$ & & 94 & 47 & 94 \\
\hline$D_{201}, D_{021}$ & & 94 & 59 & 119 \\
\hline$D_{102}, D_{012}$ & & 94 & 87 & \\
\hline$D_{211}, D_{121}$ & & 103 & 63 & 126 \\
\hline$D_{112}$ & & 103 & 89 & \\
\hline$D_{221}$ & & 126 & 73 & 145 \\
\hline$D_{212}, D_{122}$ & & 126 & 96 & \\
\hline$e_{\max } \%$ & 6.073 & 2.563 & 4.647 & 4.644 \\
\hline
\end{tabular}

The variations of scaling factor bounds $N_{\max }$ and $N_{\min }$ as functions of parameter $P$, for $5 \times 5 \times 5$ and $5 \times 5 \times 3$ distance operators are presented in Figures 6 and 7

In Table 3 we give the best integer approximation of some 3D cubic operators for $W=H=P=1$ and non cubic operators for $W=H=1$ and $P=2$. We consider $\operatorname{Dim}=512$ and $i=16$ bits. Scaling factors $N$ and maximum absolute normalized errors $e_{\max }$ are given for each operator. 
From Table 3, it can be seen that the maximum error obtained by these integer operators is close to the theoretical one (obtained with real operators of identical sizes). We notice that we have the same maximum error with an operator of size $5 \times 5 \times 3$ as that obtained with a $5 \times 5 \times 5$ operator, in the case $W=H=1$ and $P=2$.

The Distance Transform should be semi-regular [6]. Operators given in Table 3 satisfy these conditions.

\section{Conclusion}

In this paper, anisotropic local distance operators in parallelepipedic grids have been studied. These operators are useful for the analysis of 3D images such as those obtained by means of confocal microscopy or computed tomography where the sampling steps differ according to the axes.

According to the Mathematical Morphology approach, grey level images can be regarded as 3D objects in a 3D volume. Hence, 3D local distance operators turn out to be useful tools for various purposes such as image comparison.

Though exact Euclidean Distance operators are now available, Chamfer Distance operators are still of interest, especially for shape analysis, shape coding applications, and in 3D spaces.

By means of an appropriate optimization procedure, the same performances as those obtained by isotropic MxMxM operators can be achieved with lower computational complexity. Formulas providing optimal coefficients have been given for any mask size $\mathrm{M}$ and voxel elongation ratio $P / W$. Local distance operator performances have been studied for various mask sizes and voxel elongation ratio. The paper proposes a method to calculate lower and upper bounds for scaling factors in order to obtain integer approximation for the coefficients. This approach helps the algorithm perform in scenarios where the memory is limited.

\section{References}

1. Borgefors, G.: Applications using distance transforms. In: Arcelli, C., Cordella, L.P., Sanniti di Baja., G. (eds.) Aspects of Visual Form Processing, pp. 83-108. World Scientific, Singapore (1994)

2. Borgefors, G.: Distance transformations in arbitrary dimensions. Computer Vision, Graphics and Image Processing 27, 312-345 (1984)

3. Borgefors, G.: Distance transformations in digital images. Computer Vision, Graphics and Image Processing 34, 344-371 (1986)

4. Cuisenaire, O., Macq, B.: Fast Euclidean distance transformation by propagation using multiple neighborhood. Computer Vision and Image Understanding 76, 163-172 (1999)

5. Maurer Jr., C.R., Qi, R., Raghavan, V.: A linear time algorithm for computing exact Euclidean distance transforms of binary images in arbitrary dimensions. IEEE Transactions on Pattern Analysis and Machine Intelligence 25(2), 265-270 (2003)

6. Svensson, S., Borgefors, G.: Digital distance transforms in 3D images using information from neighbourhoods up to $5 \times 5 \times 5$. Computer Vision and Image Understanding 88, 24-53 (2002) 
7. Fouard, C., Malandain, G.: 3-D chamfer distances and norms in anisotropic grids. Image and Vision Computing 23, 143-158 (2005)

8. Fouard, C., Strand, R., Borgefors, G.: Weighted distance transforms generalize to modules and their computation on point lattices. Pattern Recognition 40, 2453-2474 (2007)

9. Hulin, J., Thiel, E.: Chordal axis on weighted distance transforms. In: Kuba, A., Nyúl, L.G., Palágyi, K. (eds.) DGCI 2006. LNCS, vol. 4245, pp. 271-282. Springer, Heidelberg (2006)

10. Verwer, B.: Local distances for distance transformations in two and three dimensions. Pattern Recognition Letters 12, 671-682 (1991)

11. Coquin, D., Bolon, P.: Discrete distance operator on rectangular grids. Pattern Recognition Letters 16, 911-923 (1995)

12. Remy, E., Thiel, E.: Optimizing 3D chamfer mask with norm constraints. In: Proceedings of International Workshop on Combinatorial Image Analysis, Caen, France, pp. 39-56 (2000)

13. Strand, R.: Weighted distances based on neighbourhood sequences. Pattern Recognition Letters 28(15) (2007)

14. Borgefors, G.: On digital distance transforms in three dimensions. Computer Vision and Image Understanding 64, 368-376 (1996)

15. Borgefors, G.: Weighted digital distance transforms in four dimensions. Discrete Applied Mathematics 125, 161-176 (2003)

16. Kiselman, C.: Regularity properties of distance transformations in image analysis. Computer Vision and Image Understanding 64, 390-398 (1996)

17. Sintorn, I.M., Borgefors, G.: Weighted distance transforms in rectangular grids. In: 11th International Conference on Image Analysis and Processing, Palermo, Italy, pp. 322-326 (2001)

18. Svensson, S., Borgefors, G.: Distance transforms in 3D using four different weights. Pattern Recognition Letters 23, 1407-1418 (2002)

19. Sintorn, I.M., Borgefors, G.: Weighted distance transforms for images using elongated voxel grids. In: Proc. 10th Discret Geometry for Computer Imagery, Bordeaux, France, pp. 244-254 (2002)

20. Chehadeh, Y., Coquin, D., Bolon, P.: A generalization to cubic and non cubic local distance operators on parallelepipedic grids. In: Proc. 5th Discret Geometry for Computer Imagery, pp. 27-36 (1995) 